\title{
Analysis of Mechanical Oscillations by Speckling
}

\author{
H. J. Tiziani
}

\begin{abstract}
The application of the speckle phenomenon to the analysis of in-plane translations and oscillations is first reviewed briefly. Then a practical method of investigating out-of-plane rotations (tilts) even in the presence of in-plane movements is studied. This goal can be achieved by recording the speckle patterns in the Fourier transform plane before and after the tilt or as a time-average exposure for an oscillating object. Young's fringes related to the tilts are observed in the Fraunhofer diffraction pattern when the developed photographic plate is illuminated with coherent light. This leads to a very simple engineering tool for the analysis of movements. Theoretical and experimental results will be shown.
\end{abstract}

\section{Review of Previous Work}

An optically rough surface illuminated with highly coherent light, such as from a laser, leads to a speckled appearance everywhere in space. The main features of the speckle patterns in the Fourier transform plane as well as in the image were investigated recently by a number of authors. ${ }^{1-4}$

The application of the speckle phenomenon for the measurement of in-plane translations and rotations was investigated lately. ${ }^{5-12}$ In addition, this technique was found convenient for the investigation of in-plane oscillations. ${ }^{10-12}$ Vibratory movements perpendicular to the object surface were studied by the observation of the contrast reduction in the speckle patterns. ${ }^{13-15} \mathrm{An}$ object surface with a given roughness leads to a particular speckle pattern in the Fourier transform plane as well as in the image plane. The smallest dimensions of the individual speckle in the image and in the Fourier transform plane are inversely proportional to the lateral dimensions of the lens aperture and the illuminated object field, respectively. ${ }^{1-4}$ Let us consider the surface itself to be characterized by the coherent speckle pattern generated by illuminating it with laser light. To simplify the argument this speckle pattern may be considered to be attached to the optically rough object. Photographing the speckle patterns before and after the movement of the surface has taken place will lead to two practically identical, but shifted speckle patterns from which the in-plane translation or rotation can be derived by analyzing its optical diffraction effect.

It was shown ${ }^{6-11}$ that by recording two identical, but

The author is with the Institute of Applied Physics, Swiss Federal Institute of Technology (ETH), 8049 Zürich, Switzerland.

Received 29 February 1972. shifted speckle patterns, by double exposure in the image plane, for instance, Young's fringes are obtained in its Fraunhofer diffraction pattern when illuminated with coherent light. The focal plane of the lens where the fringes are studied is referred to as the Fraunhofer diffraction plane (FDP). The fringe spacing is inversely proportional to the translation of the speckle pattern. In hologram interferometry, the amplitude of vibration of a surface (usually in the direction of the perpendicular) can be measured by applying the wellknown time-average technique, ${ }^{16}$ in which a single exposure is made while the surface is harmonically oscillating. A corresponding technique can be used for measuring vibratory motions by speckle photography. A single exposure is recorded in the image plane for inplane oscillations even in the presence of small out-ofplane movements.

The intensity distribution obtained in the FDP of the image plane, where the speckle patterns before and after the movements or as a time-average exposure (exposure over a number of oscillations) were recorded, is given by the square modulus of the autocorrelation function of the exit pupil of the lens forming the image of the object multiplied by

(1) a (cosine $)^{2}$ function for a double exposure with an in-plane translation between the exposures,

(2) a (zero-order Bessel function) ${ }^{2}$ for a time-average recording of a harmonically oscillating object or image,

(3) a $(\sin x / x)^{2}$ for a time-average recording for a translation with continuous velocity.

From the fringe spacing $p$ we obtain the in-plane translation $\Delta \xi$ and rotation as well as the amplitude of oscillation $\xi_{0}$ :

$$
\Delta \xi=\lambda f_{1} / M p, \quad \xi_{0}=0.76\left(\lambda f_{1} / M p_{1}\right),
$$

where $f_{1}$ is the focal length of the lens or the distance between the developed photographic plate and the focal 


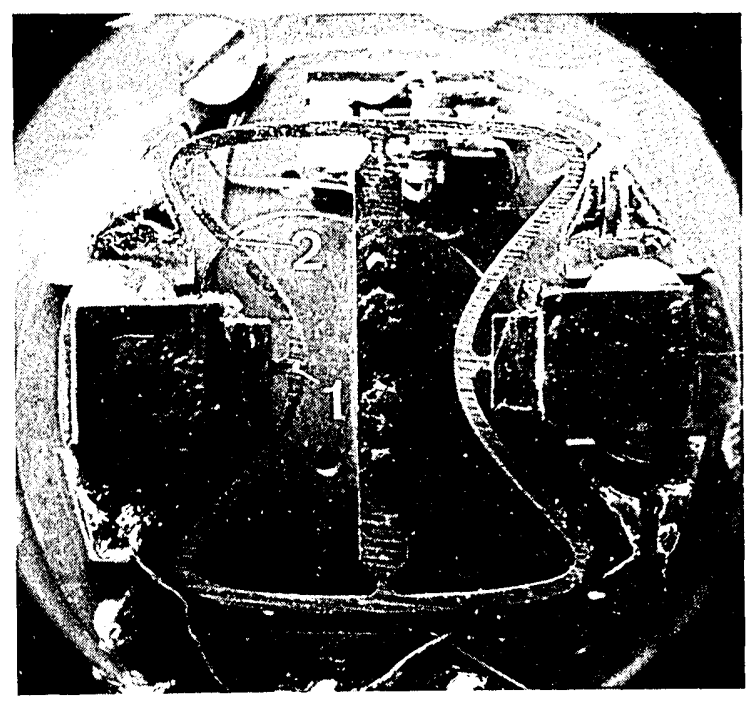

Fig. 1. Photograph of the tuning fork.

plane depending on the particular arrangement for obtaining the Fraunhofer diffraction pattern, $M$ is the lateral magnification, and $\lambda$ is the wavelength of the light used in vacuum. The separation from the origin to the first minimum of the Bessel function of order zero (and first kind) is denoted by $p_{1} / 2$.

Since the interference fringes are perpendicular to the direction of the displacement, the orientation (azimuth), but not the direction of the translation, can be determined. If the movements for the different regions of the object are different in amplitude and phase, the entire object can be recorded before and after the inplane movements or as a time-average exposure, and the developed photographic plate can be illuminated afterwards, point by point, with an appropriately narrow laser beam to select the region of constant movement. The fringes are then to be studied in the FDP. The stress of a material can be derived by measuring the change of width of the fringes from neighboring portions of the object.

The tuning fork of an electronic watch is introduced in Fig. 1. Examples of the fringes displayed in the FDP of the speckle patterns recorded in the image plane are shown in Fig. 2. The corresponding regions of the tuning fork are marked in Fig. 1. The experimental details will be given in Sec. III.

Small in-plane translations (amplitude and azimuth) of an oscillating object can also be measured. The time-average speckle pattern before and after the translation can be recorded in the image plane. In the FDP two fringe systems are obtained. The (cosine $)^{2}$ and the $J_{0}^{2}$ fringes are superimposed, leading to the analysis of the two movements. A typical example is demonstrated in Fig. 3, where the (Bessel function of order zero) ${ }^{2}$ was modulated by the (cosine) ${ }^{2}$ fringes (two dark fringes symmetrical to the center).

Small movements orthogonal to the object surface and tilts lead to a reduction in the contrast of the fringes obtained. It should further be noted that the absolute phase of the light illuminating the object is not important in the above application, and the requirements on the temporal and spatial coherence of the light used are rather relaxed compared with those of holographic techniques. However, the in-plane movements to be measured should be larger than the smallest speckle size in the object space (given by the aperture of the image-forming system) and not larger than, say, one-tenth of the lateral dimension of the object. For the analysis of very small movements the holographic techniques are the more sensitive than the speckle methods. A moderately high aperture lens is needed for recording the speckle patterns to obtain a number of fringes with good contrast in the FDP.

\section{Study of Diffraction of Speckle Patterns Recorded in Fourier Transform Plane of Object}

Holographic techniques suitable for the study of three-dimensional movements were analyzed recently by a number of authors. The interpretation of the fringes in hologram interferometry is usually rather difficult for a nonexpert, especially when larger amounts of tilts (rotations with the axis of rotation orthogonal to the line of sight) occur in the presence of considerable in-plane translations. Some of these problems are overcome in the present study where the speckle patterns are recorded in the Fourier transform plane of the object before and after a tilt or as a time-average exposure for an oscillating object.

The novel feature of the investigation reported here is that the shift of the speckle pattern in the Fourier

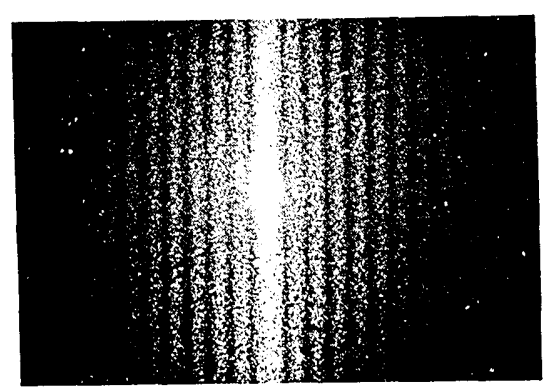

(a)

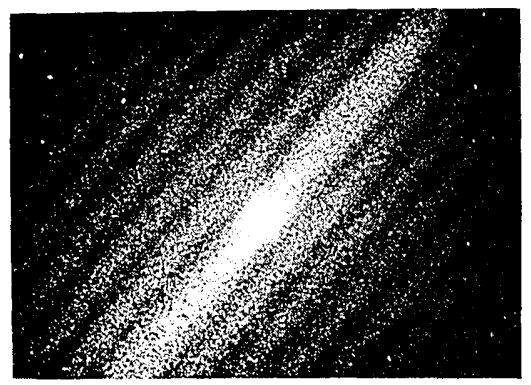

(b)

Fig. 2. Photographs of the fringes obtained in the FDP of a time-average exposure of a speckle pattern of the tuning fork recorded in the image plane (a) at the center of the symmetrical tuning fork where $p_{01}=9.5 \mu \mathrm{m}$, (b) at position 2 where $\rho_{02}=5.4 \mu \mathrm{m}$. 


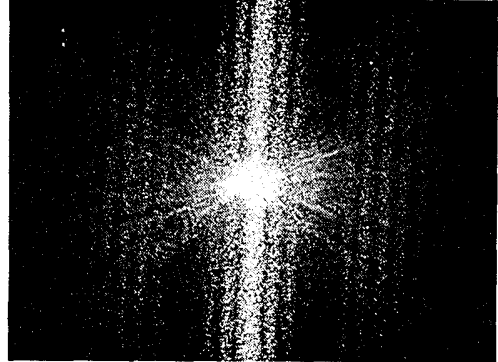

Fig. 3. Fringes displayed in the FDP when the central portion of the image with speckle patterns was illuminated. The image was recorded in the time average before and after a lateral displacement of $5 \mu \mathrm{m}\left(\rho_{01}=9.5 \mu \mathrm{m}\right)$.

transform plane is due to a tilt in another plane, the object plane. A tilt of an angle $\gamma$ introduced into the object rotates the speckle pattern, leaving the object as a whole by an angle, which is $2 \gamma$ for coherent light incident and scattered approximately along the surface normal, i.e., the surface acts like a mirror. An oblique illumination, however, is usually more convenient for practical applications. A rotation of the original speckle pattern through a different angle depending on the angle of incidence of the light was found.

We assume that the shift of the speckle pattern resulting from a tilt of the object is larger than the smallest speckle dimension. Let the optically rough object surface described by $A(\xi)$ be illuminated by a plane wave incident at an angle $\beta$ as shown in Fig. 4. The amplitude on the object may be written as

$$
A_{0}(\xi)=A(\xi) \exp [i(2 \pi / \lambda) \xi \sin \beta] .
$$

A tilt of an angle $\gamma$ introduces a phase change into the original wavefront as will be shown. The one-dimensional notation is used to simplify the writing. The axis of rotation at $H$ is orthogonal to the line of sight (optical axis) as shown in Fig. 4 . The point $P_{1}$ moves to $P_{2}$ after a tilt $\gamma$ resulting in an optical path change of $\left[Q P_{2}\right]$. Assuming the refractive index in air to be 1, we have

$$
\begin{array}{r}
{\left[Q P_{2}\right]=2\left(\xi-\xi_{0}\right) \sin (\gamma / 2) \cos [\beta-(\gamma / 2)]} \\
\approx\left(\xi-\xi_{0}\right) \gamma \cos \beta
\end{array}
$$

for small $\gamma$ 's. In our model it is assumed that, at least for small angles of tilt, the relative phase variations due to the roughness of the surface remain practically unchanged, but rotated by an angle $\gamma$, so that the complex amplitude just in front of the tilted object is

$A(\xi) \exp \{i 2 \pi \xi(\sin \beta / \lambda)] \exp [2 \pi(\gamma / \lambda) \xi]$

$$
\times \exp \left[i 2 \pi\left(\xi-\xi_{0}\right)(\gamma / \lambda) \cos \beta\right] .
$$

The laterally shifted speckle pattern in the Fourier transform plane can therefore be written as

$$
a[x-(\sin \beta / \lambda)-(\gamma / \lambda)(1+\cos \beta)] \exp \left[i 2\left(\pi \xi_{0} / \lambda\right) \gamma \cos \beta\right],
$$

where $x=X / \lambda f$, with $X=$ coordinate in Fourier transform plane (Fig. 4) and $f=$ focal length of the lens used to perform the Fourier transform of the object, $a(x)=$ Fourier transform of $A(\xi)$.

The intensity $i(x)$ recorded in the Fourier transform plane of the doubly exposed speckle patterns before and after a tilt is therefore

$$
\begin{aligned}
i_{1}(x)=|a[x-(\sin \beta / \lambda)]|^{2} & \\
& +|a[x-(\sin \beta / \lambda)-(\gamma / \lambda)(1+\cos \beta)]|^{2} .
\end{aligned}
$$

For a harmonically oscillating object illuminated by a single plane wave incident at an angle $\beta$, the time-average intensity photographed in the Fourier transform plane can be written as

$\left\langle i_{2}(x)\right\rangle_{t}=\left\langle\mid a\left[x-(\sin \beta / \lambda)-(1+\cos \beta)\left(\gamma_{0} / \lambda\right) \cos 2 \pi \nu t\right]{ }^{2}\right\rangle_{t}$,

where $\nu$ is the frequency of oscillation and $\gamma_{0}$ the maximum angular tilt (rotation with the axis of rotation perpendicular to the line of sight). For an intuitive argumentation it should be noted that the laterally oscillating speckle pattern in the Fourier transform plane remains almost stationary for a short time near the extreme positions. After photographic development of the plate, which is either doubly exposed or recorded as a time-average exposure, another Fourier transform in coherent light is performed to obtain the fringes in the FDP :

$$
A^{\prime}\left(u^{\prime}\right)=\int_{\infty}^{\infty} T(x)\left(\exp i 2 \pi u^{\prime} x\right) d x,
$$

where $T(x)$ is the amplitude of transmission of the developed photographic plate and $u^{\prime}=\xi^{\prime} f / f_{1}$, with $\xi^{\prime}=$ coordinate in the FDP and $f_{1}=$ focal length of the second lens or distance between the plate and focal plane, as pointed out in the first section.

By considering the amplitude transmittance of the photographic material to be proportional to the recorded intensity, ${ }^{17}$ the intensity in the FDP can be written for the doubly exposed plate (before and after a tilt $\gamma$ ),

$$
\begin{aligned}
I_{1}^{\prime}\left(u^{\prime}\right)=2 \mid A^{\prime}\left(u^{\prime}\right) \otimes & \left.A^{\prime}\left(-u^{\prime}\right)\right|^{2} \\
& \times\left\{1+\cos \left[2 \pi u^{\prime}(\gamma / \lambda)(1+\cos \beta)\right]\right\},
\end{aligned}
$$

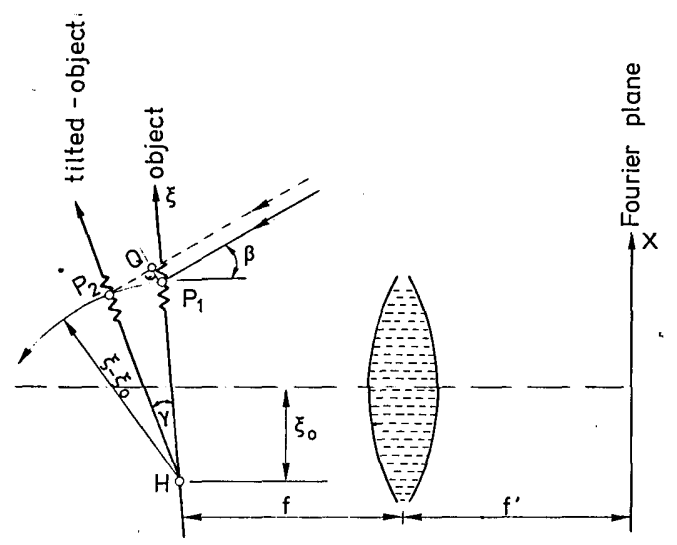

Fig. 4. Arrangement to record the speckle patterns in the Fourier transform plane. 
and for the time-average recording of the harmonically oscillating object, for an exposure time $t_{0} \gg 1 / \nu$

$$
I_{2}{ }^{\prime}\left(u^{\prime}\right)=\left|A^{\prime}\left(u^{\prime}\right) \otimes A^{*}\left(-u^{\prime}\right)\right|^{2} J_{0}{ }^{2}\left[2 \pi\left(\gamma_{0} / \lambda\right) u^{\prime}(1+\cos \beta)\right] .
$$

Since $A^{\prime}\left(u^{\prime}\right)$ is the Fourier transform of $a(x)$ and $\otimes$ indicates convolution and $J_{0}$ is the Bessel function of order zero and first kind, we obtain in the FDP an intensity proportional.to the square modulus of the autocorrelation of the illuminated object field, multiplied by (cosine) ${ }^{2}$ for a tilt or the (zero-order Bessel function $)^{2}$ for an oscillating tilt or $\left(\sin u^{\prime} / u^{\prime}\right)^{2}$ for a continuous tilt.

From the width of the cosine fringes $\Delta \xi^{\prime}$ [in expression (7) ] the tilt angle in the object plane is found to be

$$
\gamma=\lambda f_{1} / f(1+\cos \beta) \Delta \xi^{\prime}
$$

and the maximum amplitude $\gamma_{0}$ of the angular tilt [from expression (8) ], by denoting the separation of the first minimum from the origin by $a_{1} / 2$, as

$$
\gamma_{0}=0.76 \lambda f_{1} / f(1+\cos \beta) a_{1} .
$$

An interesting observation can be made when the timeaverage speckle pattern is first recorded and then superimposed on the speckle pattern of the stationary object. This yields a modified fringe pattern in the FDP, namely,

$$
\begin{aligned}
I_{3}\left(u^{\prime}\right)=\left|A^{\prime}\left(u^{\prime}\right) \otimes A^{* \prime}\left(-u^{\prime}\right)\right|^{2} & \\
& \times\left|K+J_{0}\left[2 \pi\left(\gamma_{0} / \lambda\right) u^{\prime}(1+\cos \beta)\right]\right|^{2},
\end{aligned}
$$

where $K$ is a constant depending on the illumination of the stationary and time-average speckle patterns. If $K>\left|K_{0}\right|\left(K_{0}=\right.$ amplitude of the first minimum of $\left.J_{0}\right)$ and $J_{0}^{\prime \prime}>0$, the minima for $I_{3}{ }^{\prime}\left(u^{\prime}\right)$ appear for $J_{0}{ }^{\prime}=0$. This result may sometimes be useful when larger movements are analyzed with the same experimental configuration; examples are shown in Fig. 7(d) and (e).

\section{Experimental Arrangement and Procedure}

In our applications an $\mathrm{He}-\mathrm{Ne}$ laser of $18-\mathrm{mW}$ power with a wavelength of $632.8 \mathrm{~nm}$ was used as a light source. For convenience, Agfa-Gevaert Scientia 10E75 plates and films were chosen for registration of the speckle patterns. They have the necessary fine grain and give minimum grain noise scatter. The exposure times were of the order of a few seconds. No special care was needed during the processing of the plates and films to obtain fringes of good contrast. No additional treatment of the surface such as painting was needed. For the analysis of the in-plane movements the speckle patterns were recorded in the image plane with a lateral magnification $M=-3$ and $f$ ratio $f / 2.8$. Most of the work presented was carried out to study the mechanical oscillations (in-plane and tilts) of the tuning fork of a new design of an electronic watch (Fig. 1). The resonant frequency of oscillation was $1050 \mathrm{~Hz}$. The total length of the tuning fork was $20 \mathrm{~mm}$. The fringes obtained in the FDF from the lateral in-plane movements are displayed in Fig. 2 for the appropriate position of the tuning fork. They result from the time-

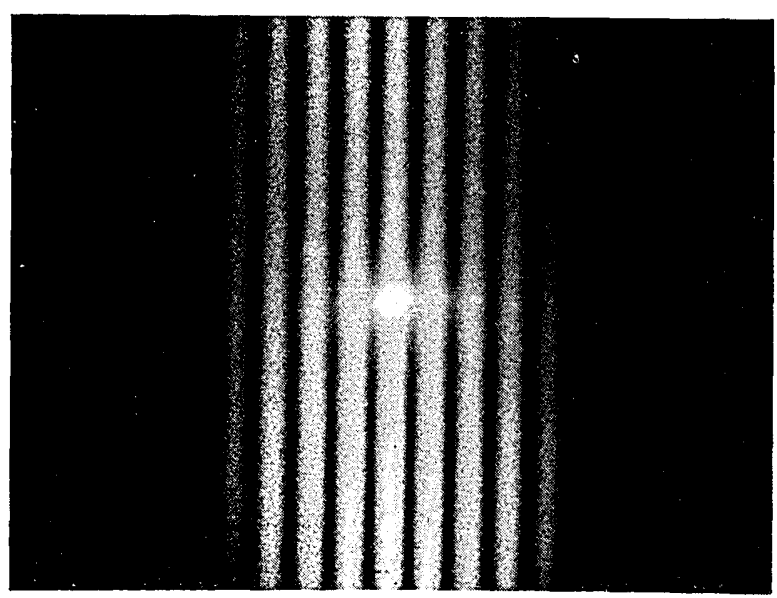

(a)

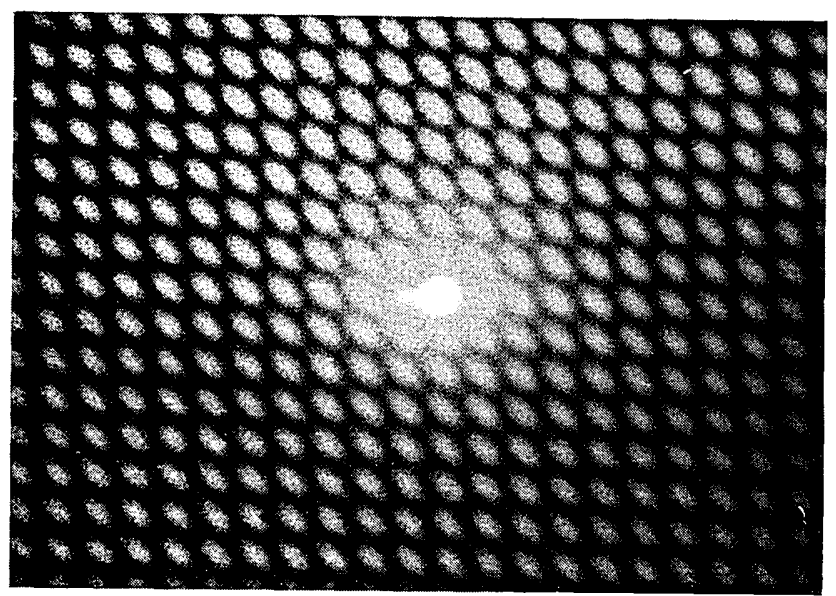

(b)

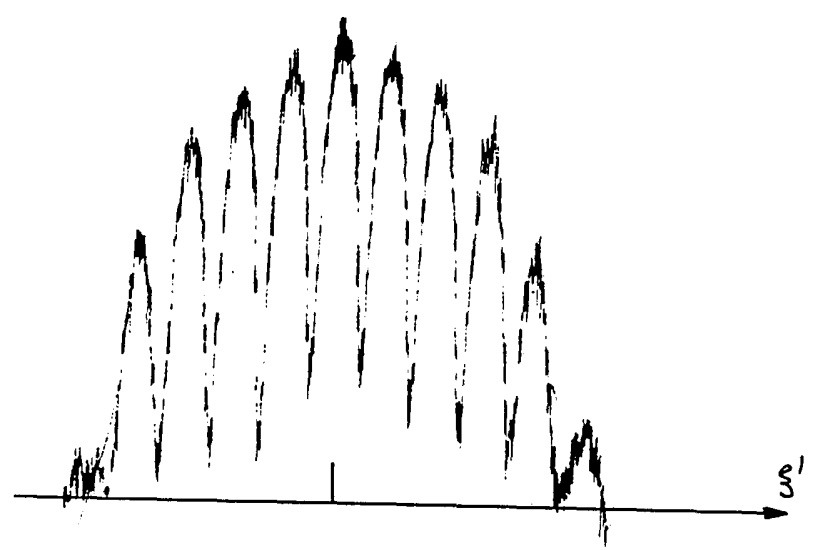

(c)

Fig. 5. Photographs of the fringes obtained in the FDP from the speckle patterns recorded in the Fourier transform plane of an Al plate, illuminated by a plane wave $\left(\beta=45^{\circ}\right)$. (a) Doubly exposed speckle patterns with a tilt $\gamma_{\xi}=60 \mathrm{sec}$ of arc introduced between the two exposures $(\gamma \xi \equiv \gamma$ in Fig. 4), (b) two doubly exposed speckle patterns with a tilt $\gamma_{\xi}=60 \mathrm{sec}$ of arc between the first and second exposure and a tilt $\gamma_{\eta}=60 \mathrm{sec}$ of arc perpendicular to $\gamma \xi$ between the second and third exposure, (c) microdensitometer trace of (a) slightly below the center. 


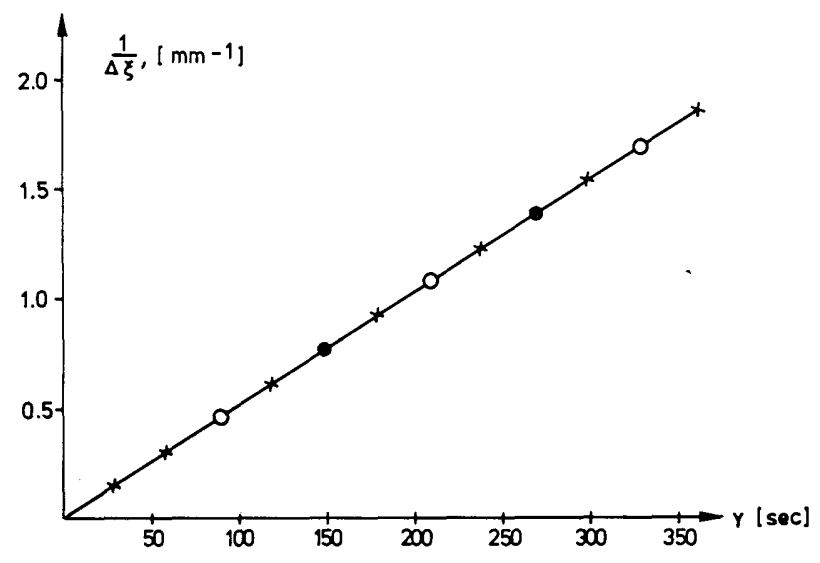

Fig. 6. The inverse of the fringe spacings obtained in the FDP is plotted against the tilt angle for tilts $\gamma_{\xi}(\gamma \xi \equiv \gamma$ in Fig. 4) and tilts $\gamma_{\eta}$ perpendicular to $\gamma \xi$ marked by 0 . The results for tilts $\gamma \xi$ with an additional translation of $40 \mu \mathrm{m}$ are marked by $\otimes$. $\left(\beta=45^{\circ}\right)$.

average recording of the speckle patterns in the image plane.

The experimental arrangement for the study of tilts is shown in Fig. 4. In addition a jig was designed to introduce known tilts into the object illuminated by a plane wave. For comparison, the tilts introduced were measured very accurately by autocollimation. It should be noted that the axis of rotation must not coin- cide with the object surface under test, as pointed out in Ref. 18. The developed photographic plates and films were then illuminated by a converging spherical wave from the $\mathrm{He}-\mathrm{Ne}$ laser, and the fringes were studied in the rear focal plane (FDP).

Figure 5 shows the fringes obtained in the FDP from a doubly exposed small Al plate (lateral width $6 \mathrm{~mm}$ ), where a tilt $\gamma_{\xi}=60 \mathrm{sec}$ of arc was introduced between the two exposures. A plane wave was used to light the object at an angle $\beta=45^{\circ}$. The tilt fringes in the FDP for two doubly exposed speckle patterns of a larger metallic surface are displayed in Fig. 5(b), where a tilt $\gamma_{\xi}$ of $60 \mathrm{sec}$ of arc was introduced between the first and the second exposure ( $\gamma_{\xi}=\gamma$ in Fig. 4) and a tilt $\gamma_{\eta}$ of $60 \mathrm{sec}$ of arc perpendicular to $\gamma_{\xi}$ was added before the third exposure. The two fringe systems are equidistant and perpendicular to each other. In addition, the fringes that would occur by introducing $\gamma_{\xi}$ and $\gamma_{\eta}$ in turn, before and after a double exposure, are also visible. In Fig. 5(c) a microdensitometer trace recorded slightly outside the central portion of Fig. 5(a) is shown. The results of a number of experiments where known tilts $\gamma_{\xi}$ and $\gamma_{\eta}$ were introduced are presented in Fig. 6, where the inverse of the fringe spacings $1 / \Delta \xi$, is plotted, against the angle of tilt. In-plane translations of 40 $\mu \mathrm{m}$ were added to the tilt in some experiments. They had, however, no consequences on the results obtained, as shown by the points marked $\otimes$ in Fig. 6 . Therefore, tilts can be measured accurately even in the presence of small in-plane translations. The results for different

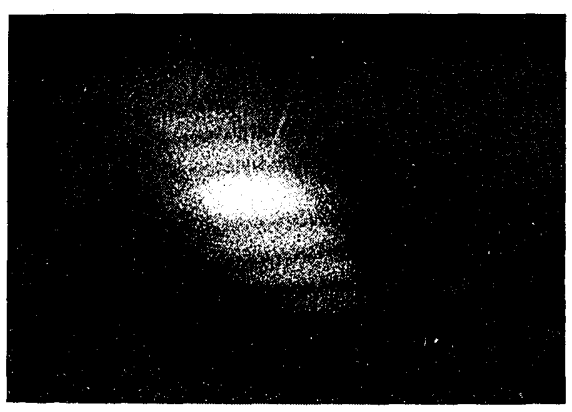

(a)

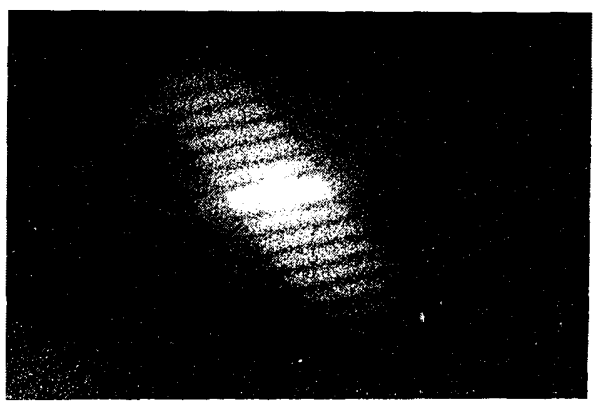

(b)

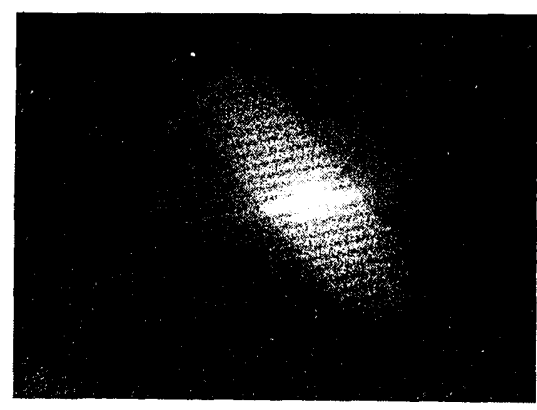

(c)

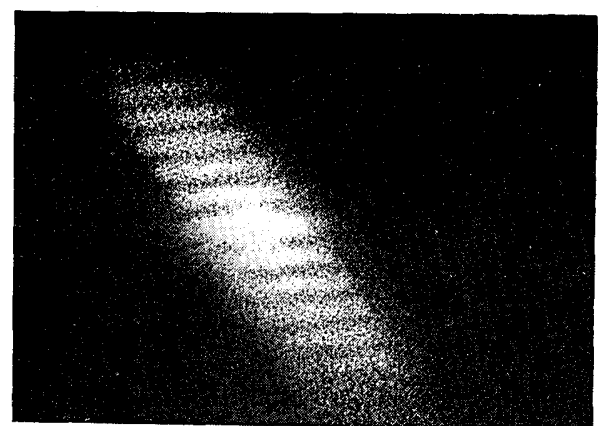

(d)

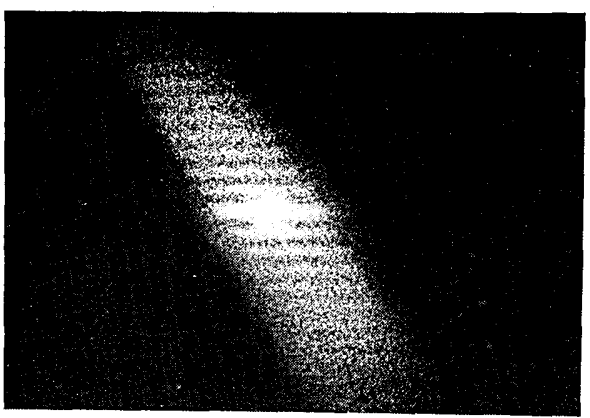

(e)

Fig. 7. Photographs of the fringes displayed in the FDP for the time-average speckle patterns recorded in the Fourier transform plan of region 2 in Fig. 1. The resonant frequency was about $1050 \mathrm{~Hz}$, and the voltages applied to the coils were $U=1.0 \mathrm{~V}, 1.5 \mathrm{~V}$, and $2.5 \mathrm{~V}$ for Fig. 7(a), (b), and (c) yielding tilts in the azimuth perpendicular to the fringes; (d) and (e) show fringes obtained in the FDP of the doubly exposed speckle patterns (stationary and time-average) for $U=2.5 \mathrm{~V}$ and $3.5 \mathrm{~V}$. 

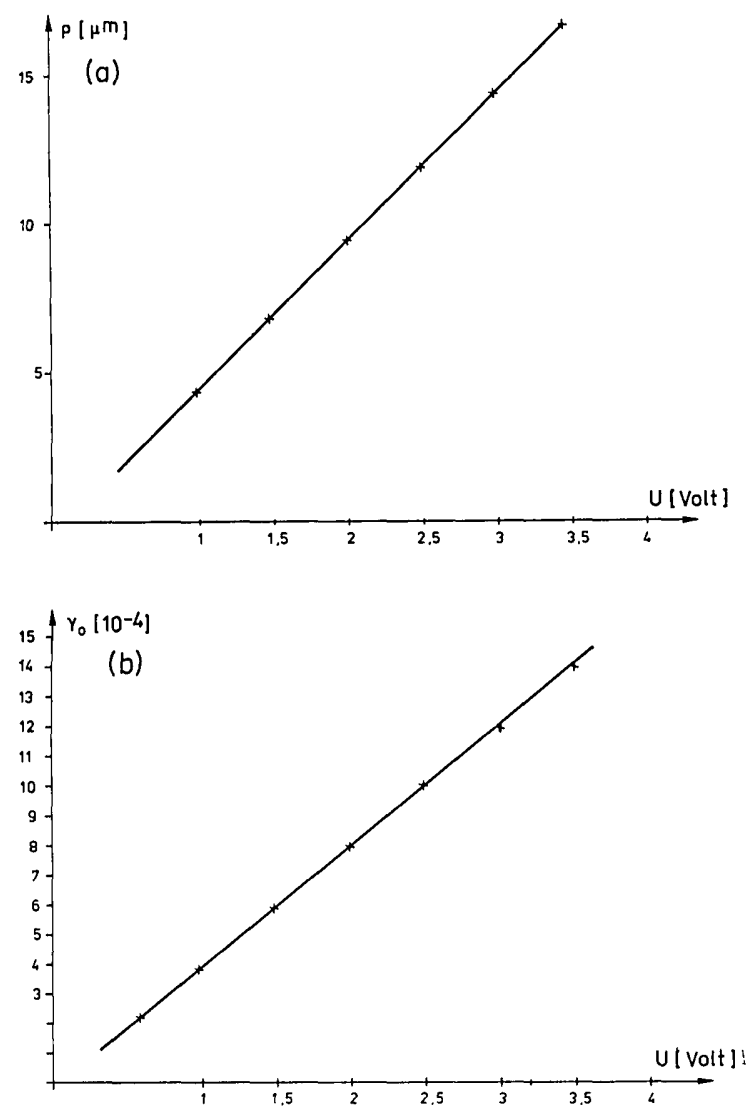

Fig. 8. Amplitudes of the mechanical oscillations as function of the voltages applied for (a) in-plane movements of the central portion 1 and (b) tilts $\gamma_{0}$ of position 2 .

incident angles and for one- and two-beam illumination are given elsewhere. ${ }^{18}$ Very good agreement was obtained between the theory based on the proposed model and the experiments.

For the measurements of the rotational oscillations (tilts) even in the presence of a lateral oscillation, the time-average speckle pattern was recorded in the Fourier transform plane. The corresponding fringes in the FDP are shown in Fig. 7(a), (b), and (c) for different voltages applied to the coils of the electromagnetic head. By contrast, the fringes obtained in the FDP from the superposition of the speckle patterns of the stationary object on that of the time-average recording are shown in Fig. 7(d) and (e) for higher voltages (larger tilts). The exposure for the stationary object was chosen to be half that of the oscillating one, namely, $2 \sec (f=$ $50 \mathrm{~mm}, f / 4$, and $f_{1}=120 \mathrm{~mm}$ ).

The amplitudes of the lateral in-plane mechanical oscillations and tilts of the tuning fork (Fig. 1) are plotted in Fig. 8. As a consequence of the results a further study of the shape of the tuning fork may be carried out to reduce the tilts. Results obtained by holographic techniques of the tuning forks are presented in Ref. 11.

For some of our special applications it was found convenient to apply two-beum illumination and record the time-average speckle pattern in the image plane. A fringe pattern obtained this way is shown in Fig. 9. The two bright fringes symmetrical to the central fringe indicate an out-of-plane movement in the presence of an in-plane oscillation of the tuning fork near 2 in Fig. 1. A detailed analysis of the three-dimensional movements with two-beam illumination will be given in a separate publication. By contrast with one-beam illumination the fringes of Fig. 2(b) were obtained.

The fringes in the FDP of a time-average speckle pattern recorded in the Fourier transform plane of an oscillating quartz $(\nu=8000 \mathrm{~Hz})$ are shown in Fig. 10 .

\section{Conclusions}

The method described may become a very useful engineering tool and has many potential applications. No reference wave is needed, and the practical implementations of the analysis are not very demanding. No special processing and no sophisticated equipment is necessary to perform the investigations. A conventional camera can be used to photograph the speckle patterns. In-plane oscillations can be studied in the presence of small tilts by recording the speckle patterns in the image plane. By contrast, tilts can be measured with high accuracy, even in the presence of small inplane translations, by a double-exposure, or time-

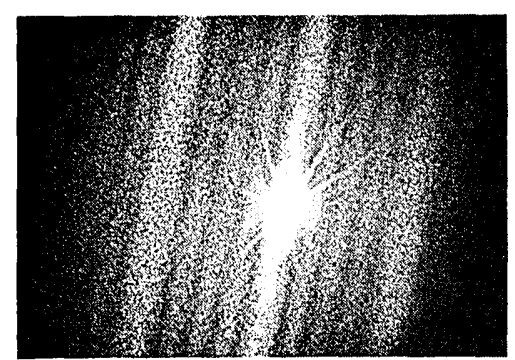

Fig. 9. Fringes in the FDP of the speckling recorded in the image plane for two incident light beams (position 2 in Fig. 1) for $U=2.5 \mathrm{~V}\left(\beta_{1} \approx-\beta_{2}\right)$.

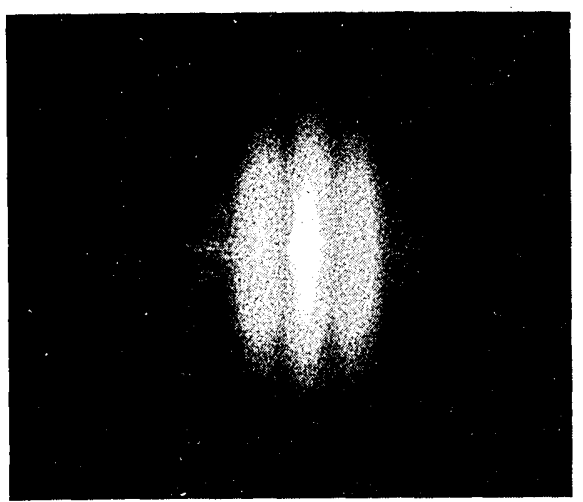

Fig. 10. Example of the fringes in the FDP obtained from a time-average speckle pattern recorded in the Fourier transform plane of an oscillating quartz. 
average, recording of the speckle patterns in the Fourier transform plane. For some applications it may be useful to reimage the recorded speckle patterns by a lens with an aperture stop offset from the axis placed in its rear focal plane to act as a filter. In this way it is possible to select the appropriate part of the motion. The method proved to be very accurate (better than $3 \%$ ) for translation as well as tilt measurements. For tilt measurements the angle of incidence of the illumination has to be known. The $3 \%$ limit was mostly imposed by the precision of our experimental equipment.

The new speckle method was found to be very convenient and accurate for our applications. The use of the speckle phenomenon for three-dimensional displacement and vibration analysis can complement holographic methods in many engineering applications. They operate best at sensitivities slightly lower than that of holographic interferometry. The requirement for our application of the speckle phenomenon is that the displacement of the speckle pattern in the appropriate plane due to a tilt of the object or a translation be greater than the smallest speckle width in the corresponding plane.

The author would like to thank the Director of the Institute of Applied Physics and the Department of Industrial Research at the ETH Zurich, E. Baumann, for support of the work. Thanks are further due to A.
Greuter for providing the tuning fork and A. Aemmer for helping with the preparation of some of the experiments.

\section{References}

1. L. I. Goldfischer, J. Opt. Soc. Am. 55, 247 (1965).

2. H. J. Tiziani, thesis, London University (1967).

3. H. H. Hopkins and H. J. Tiziani, in Proceedings of the International Symposium on Holography, Besançon (1970).

4. J. C. Dainty, Opt. Acta 17, 761 (1970).

5. J. A. Leendertz, J. Phys. E. 3, 214 (1970).

6. J. M. Burch and J. M. J. Tokarski, Opt. Acta 15, 101 (1968).

7. S. Debrus, M. Françon, and M. May, in Proceedings of ICO Conference, Reading (1969).

8. J. N. Butters and J. A. Leendertz, J. Phys. E. 4, 277 (1971).

9. E. Archbold, J. M. Burch, and A. E. Ennos, Opt. Acta 17, $883(1970)$.

10. H. J. Tiziani, Opt. Acta 18, 891 (1971).

11. H. J. Tiziani, Optik 34, 442 (1972).

12. U. Köpf, Optik 33, 517 (1971).

13. E. Archbold et al., Nature 222, 263 (1969).

14. E. Eliasson and F. M. Mottier, J. Opt. Soc. Am. 61, 559 (1971).

15. N. Fernelius and C. Tome, J. Opt. Soc. Am. 61, 556 (1971).

16. R. L. Powel and K. A. Stetson, J. Opt. Soc. Am. 55, 1593 (1965).

17. A. Kozma, J. Opt. Soc. Am. 56, 428 (1966).

18. H. J. Tiziani, Opt. Commun., July 1972.

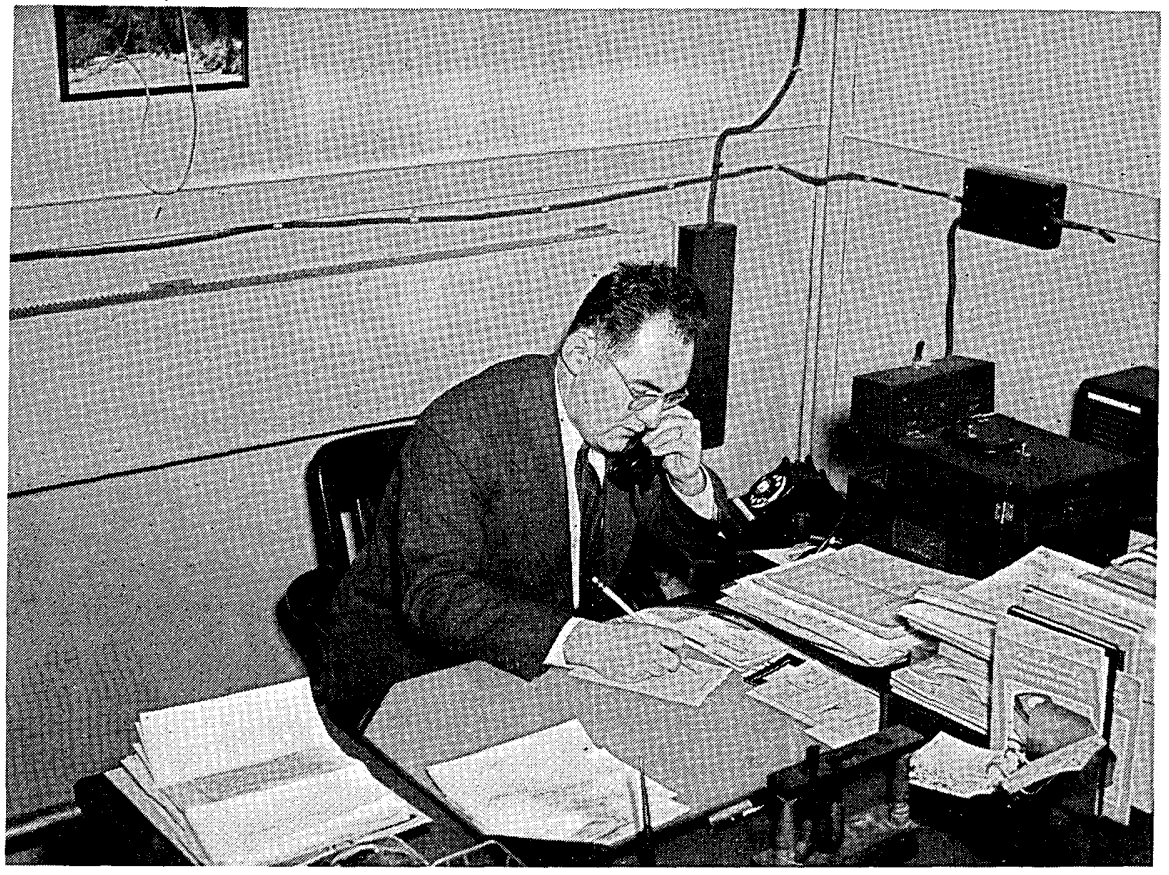

Duncan Macdonald who was director of the Boston University Optical Research Laboratory when this photograph was taken in 1946. 